\title{
Adjuvant chemotherapy for gastric cancer: a randomised phase 3 trial of mitomycin-C plus either short-term doxifluridine or long-term doxifluridine plus cisplatin after curative D2 gastrectomy (AMC0201)
}

Y-K Kang ${ }^{*}, 1$, H-M Chang ${ }^{1}$, J H Yook ${ }^{2}$, M-H Ryu ${ }^{1}$, I Park ${ }^{1}$, Y J Min ${ }^{3}$, D Y Zang ${ }^{4}$, G Y Kim ${ }^{5}$, D H Yang ${ }^{6}$, S J Jang ${ }^{7}$,

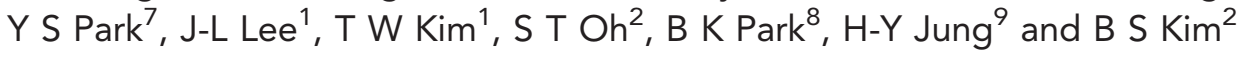

${ }^{1}$ Department of Oncology, Asan Medical Center, University of Ulsan College of Medicine, 88, Olympic-ro 43-gil, Songpa-gu, Seoul 138-736, Korea; ${ }^{2}$ Department of Surgery, Asan Medical Center, University of Ulsan College of Medicine, Seoul 138-736, Korea; ${ }^{3}$ Department of Internal Medicine, Ulsan University Hospital, University of Ulsan College of Medicine, Ulsan 682-060, Korea; ${ }^{4}$ Department of Internal Medicine, Hallym University Sacred Heart Hospital, Hallym University College of Medicine, Anyang 431070, Korea; ${ }^{5}$ Department of Surgery, Ulsan University Hospital, University of Ulsan College of Medicine, Ulsan 682-060, Korea; ${ }^{6}$ Department of Surgery, Hallym University Sacred Heart Hospital, Hallym University College of Medicine, Anyang 431-070, Korea; ${ }^{7}$ Department of Pathology, Asan Medical Center, University of Ulsan College of Medicine, Seoul 138-736, Korea; ${ }^{8}$ LSK Global Pharma Services, Seoul, Korea and ${ }^{9}$ Departments of Gastroenterology, Asan Medical Center, University of Ulsan College of Medicine, Seoul 138-736, Korea

Background: This phase 3 study evaluated the efficacy of new adjuvant chemotherapy (MFP), which intensified the mitomycin-C (MMC) plus short-term doxifluridine (Mf) for gastric cancer.

Patients and methods: A total of 855 patients (424 in Mf, 431 in MFP) with pathological stage II-IV (M0) gastric cancer after D2 gastrectomy were randomly assigned to receive either Mf (MMC $20 \mathrm{mg} \mathrm{m}^{-2}$, followed by oral doxifluridine $460-600 \mathrm{mg} \mathrm{m}^{-2}$ per day for 3 months) or MFP (MMC $20 \mathrm{mg} \mathrm{m}^{-2}$, followed by oral doxifluridine $460-600 \mathrm{mg} \mathrm{m}^{-2}$ per day for 12 months with 6 monthly infusions of $60 \mathrm{mg} \mathrm{m}^{-2}$ of cisplatin) chemotherapy.

Results: With a median follow-up of 6.6 years, there was no difference between the two groups in recurrence-free survival (RFS) (5-year RFS 61.1\% in Mf and 57.9\% in MFP; hazard ratio 1.10 (95\% Cl 0.89-1.35); $P=0.39$ ) and overall survival (OS) (5-year OS 66.5\% in Mf and 65.0\% in MFP; hazard ratio 1.11 (95\% Cl 0.89-1.39); $P=0.33$ ).

Conclusion: Intensification of Mf adjuvant chemotherapy by prolonging the duration of oral fluoropyrimidine and adding cisplatin was safe but not effective to improve the survivals in curatively resected gastric cancer patients. 
Gastric cancer (GC) is the second most common cause of cancerrelated mortality worldwide, with 989600 new cases and 738000 deaths per year (Jemal et al, 2011). For locally advanced cases, the only treatment option for potential cure is complete surgical resection. Unfortunately, a significant number of patients experience recurrence even after complete resection, and the prognosis of recurrent GC is dismal. The high recurrence rate and poor prognosis have led to extensive investigation of the use of adjuvant treatments to improve survival. However, there had been a debate on the role of adjuvant treatment until early 2000, especially in western countries, primarily due to the lack of large pivotal study, although several meta-analyses consistently suggesting small but significant benefits (Hermans et al, 1993; Earle and Maroun, 1999).

In 2000, when the AMC0201 trial was designed, adjuvant chemotherapy was popularly used in clinical practice in Korea. Because of this widespread practice, we believed that using surgery only in the control arm would not be appropriate. Thus, we selected adjuvant chemotherapy rather than surgery alone as the control arm and aimed to focus on reinforcing its efficacy in this trial. Mitomycin-C (MMC)-based regimens were one of the most popularly used adjuvant chemotherapies at that time (Chang et al, 2002; Koo et al, 2007). A Japanese meta-analysis suggested the efficacy of MMC-based adjuvant chemotherapy, and a Spanish randomised phase 3 study showed the survival benefit of simple combination of MMC and 3 months of oral fluoropyrimidine, although sample size of this study was small (Nakajima et al, 1994; Cirera et al, 1999). On the basis of these results, we decided to use MMC plus short-term oral fluoropyrimidine (Mf) as a reference treatment. To strengthen this regimen, we mapped out the following two strategies. We prolonged the duration of oral fluoropyrimidine, and also added cisplatin, an active component of effective regimens for metastatic GC (MFP).

We then performed this prospective, randomised phase 3 study to determine whether MFP could improve recurrence-free survival (RFS) over the Mf regimen in patients with GC after curative D2 resection. Here we report the final results of the AMC0201 study with inclusion of long-term outcome.

\section{MATERIALS AND METHODS}

Study design and patients. AMC0201 was an open-label, prospective randomised phase 3 clinical trial conducted at three centres in Korea.

The criteria for eligibility were histologically proven gastric adenocarcinoma; D2 lymph-node dissection; R0 resection; an age of 18-70 years; pathological stage II-IV without distant metastasis according to the 6th edition of American Joint Committee on Cancer (AJCC) staging system; an Eastern Cooperative Oncology Group (ECOG) performance status of $0-2$; and adequate renal, liver and bone marrow function. Exclusion criteria included any of the following: incomplete surgical resection, prior chemotherapy, immunotherapy, or radiotherapy, concurrent or previous malignancy within the past 5 years and positive M1 lymph nodes on postoperative pathologic examination.

The protocol was approved by the institutional review board at each participating institution and conducted in accordance with the ethical principles of the Declaration of Helsinki and also the Good Clinical Practice guidelines, as defined by the International Conference on Harmonisation. All patients provided written informed consent before entry into the study.

Study treatment. At 3 weeks after surgery, eligible pathological stage II-IV (M0) cases were randomly assigned at a 1:1 ratio to receive the Mf or MFP regimen. Randomisation was stratified according to stage and each participating centre based on a permutation block method. Patients and investigators were not masked to study treatment.

For patients randomised to the Mf group, $20 \mathrm{mg} \mathrm{m}^{-2} \mathrm{MMC}$ was given intravenously 3-6 weeks after surgery, and then 4 weeks later, daily oral doses of $460 \mathrm{mg} \mathrm{m}^{-2}$ doxifluridine were administered for 3 months. In the MFP group, $20 \mathrm{mg} \mathrm{m}^{-2}$ MMC was given intravenously 3-6 weeks after surgery, and beginning 4 weeks later, $60 \mathrm{mg} / \mathrm{m}^{-2}$ cisplatin was given intravenously monthly for 6 months and $460 \mathrm{mg} \mathrm{m}^{-2}$ per day oral doxifluridine was administered for 12 months. One cycle lasted for 1 month. The doxifluridine dose was increased to $600 \mathrm{mg} \mathrm{m}^{-2}$ per day in both groups after interim safety analysis in February 2004. Dose modification was prespecified for patients who experienced haematologic and non-haematologic toxicities (Supplementary Data).

Assessments. At baseline, patients underwent a history, physical examination, including weight, height and vital signs. Computed tomography (CT) scans of the abdomen and pelvis with contrast enhancement, chest radiography and electrocardiography were performed. In addition, laboratory tests, including complete blood count with differential counts, electrolytes, coagulation test, liver and renal function testes were obtained. Adverse events and laboratory profiles were assessed every 4 weeks during treatment. After completion of chemotherapy, clinical assessments were performed every 3 months up to 2 years from surgery, every 6 months between 2 and 5 years from surgery and every 1 year thereafter. Plain chest radiography and abdominopelvic contrastenhanced CT scans were performed every 6 months within 5 years from surgery and every 1 year thereafter; gastroscopy was performed every 1 year. If there was any sign or symptom indicating recurrence, investigations were immediately carried out to verify the status of the patients. If findings on imaging studies were suggestive but not conclusive, rigorous serial follow-up studies were performed to detect recurrence. The date of the first recognition of findings suggestive of recurrence was defined as the date of recurrence. Adverse events were graded according to the National Cancer Institute's Common Terminology Criteria for Adverse Events, version 2.0.

Statistical analysis. The primary end point was 3-year RFS, and the secondary end points were 3-year overall survival (OS), disease recurrence and safety. All randomised patients were analysed on an intention-to-treat basis. On the basis of the result of previous trials, the 3-year RFS rate in the Mf group was estimated to be $60 \%$. This trial was initially designed to identify a $15 \%$ improvement of 3 -year RFS rate in the MFP group and a total of 475 patients were required for a two-sided $\alpha$ of $5 \%$ and a statistical power of $90 \%$, considering $10 \%$ of follow-up loss.

In February 2004, when we had enroled half of the planned number of patients (245), we performed a planned interim analysis for safety monitoring and potential sample size recalculation based on the distribution of pathological stage in study population. After the study began, several participating investigators concerned that the original dose of doxifluridine was low. As the toxicity of original dose was mild and well tolerated at interim analysis, we decided to increase the doxifluridine dose from 460 to $600 \mathrm{mg} \mathrm{m}^{-2}$ per day by protocol amendment. When we analysed the distribution of pathological stage until that point, patients with early stage were included more than we originally expected. On the basis of this, the 3 -year RFS of control arm was re-estimated as better than our original assumption. This estimation and rapid patient accrual enabled us to increase the sample size to detect accurately real, even if small, improvements in efficacy. Therefore, the estimated 3-year RFS rate in the Mf group was increased to $70 \%$, while the expected additional benefit in the MFP group was lowered to $10 \%$ ( $80 \%$ of 3 -year RFS rate). With the same type I and II error rates, we estimated that a total enrolment of 881 patients 
(207 events) would be necessary for a hazard ratio (HR) of 0.6256 in the MFP group as compared with the Mf group.

Recurrence-free survival was defined as the time from randomisation to documented disease recurrence or death, and OS as the time from randomisation to death from any cause. Patients were censored if they were recurrence-free or alive at the last follow-up. Kaplan-Meier curves were used to estimate survival, and compared using the log-rank test. The Cox regression model was used to estimate HRs based on the comparison of the efficacy between the Mf and MFP arms in both primary analysis and subgroup analyses. All tests were two-sided, and a $P$-value $<0.05$ was considered to indicate statistical significance. Confidence intervals (CI) are at the 95\% level. Descriptive analyses were used to represent adverse events. The relative dose intensity (RDI) was calculated as the percentage of actually administered dose to planned dose per unit time. Statistical analyses were performed by a qualified biostatistician (BKP) using SAS version 9.1 (SAS Institute Inc., Cary, NC, USA) and SPSS version 14.0 (SPSS Inc., Chicago, IL, USA).

After 229 events occurred with a median follow-up period of 3.5 years, the database was initially locked on 20 March 2008 and planned primary analysis was performed. These results were previously presented at ASCO annual meeting, 2008 (Chang et al, 2008). Afterwards, to verify the long-term results of this study, 3year extension analysis was performed. The data cutoff for this extension analysis was 11 April 2011. Median follow-up at that time was 6.6 years (maximum 109.4 months).

\section{RESULTS}

Patient characteristics. Between February 2002 and August 2006, 855 patients were randomly allocated to study arms (424 in MF and 431 in MFP; Figure 1). Baseline characteristics turned out to be well balanced between the two groups (Table 1). Median time interval between surgery and chemotherapy was 23 days in the Mf group and 24 days in the MFP group. Postoperative stages were II in $51 \%$, IIIA in $31 \%$, IIIB in $9 \%$ and IV in $9 \%$ of patients. All patients received D2 surgery with R0 resection.
Treatment delivery. Planned treatment was completed in 93\% of the Mf group and $72 \%$ of the MFP group. Throughout the treatment period, 145 patients (34\%) underwent dose reduction for doxifluridine in the Mf group compared with 322 (75\%) and 19 (4\%) for doxifluridine and cisplatin in the MFP group. The reasons for dose reduction were haematologic toxicities $(97 \%$ in the Mf group; $97 \%$ for doxifluridine and $42 \%$ for cisplatin in the MFP group), or non-haematologic toxicities (3\% in the Mf group; 3\% for doxifluridine and $58 \%$ for cisplatin in the MFP group). Dose delay was applied in 64 patients (15\%) in the Mf group and 189 patients (44\%) in the MFP group, mainly because of haematologic toxicities ( $83 \%$ in the Mf group and $86 \%$ in the MFP group) of all adverse events. Median RDI per cycle for doxifluridine was $96.7-100.0 \%$ in the Mf group and $79.0-100.0 \%$ in the MFP group. The median RDI per cycle for cisplatin was $100.0 \%$ throughout all cycles.

Safety. Adverse events that occurred in $10 \%$ or more of patients are summarised in Table 2. The population evaluated for safety comprised 847 patients (422 in the Mf group and 425 in the MFP group), with the exclusion of the patients who did not receive allocated treatment after randomisation or were lost to follow-up after MMC administration. Both regimens were well tolerated, and the adverse events of all grades were more frequent in patients who received MFP than in those who received Mf. Grade 3 or 4 neutropenia was more common in the MFP group (35\%) than in the Mf group (11\%). Two patients in the Mf group and four patients in the MFP group developed febrile neutropenia. Grade 3 or 4 thrombocytopenia was rare and similar in both arms (3\% in both groups). Grade 3 or 4 non-haematologic adverse events were uncommon in both groups; however, patients in the MFP group had slightly higher rates of fatigue, anorexia, nausea, vomiting and diarrhoea. There was no treatment-related death. Between patients enroled before and after the introduction of protocol amendment in each Mf and MFP groups, there were no significant differences in the frequency of grade 3 or 4 haematologic or non-haematologic toxicities, except that grade 3 or 4 neutropenia in the MFP group was significantly increased with higher dose of doxifluridine $(22 \%$ vs $14 \% ; P=0.003)$. Actually administered dose of doxifluridine during six cycles of study treatments was increased after the protocol amendment than before (mean 517.89 vs $409.24 \mathrm{mg} \mathrm{m}^{-2}$

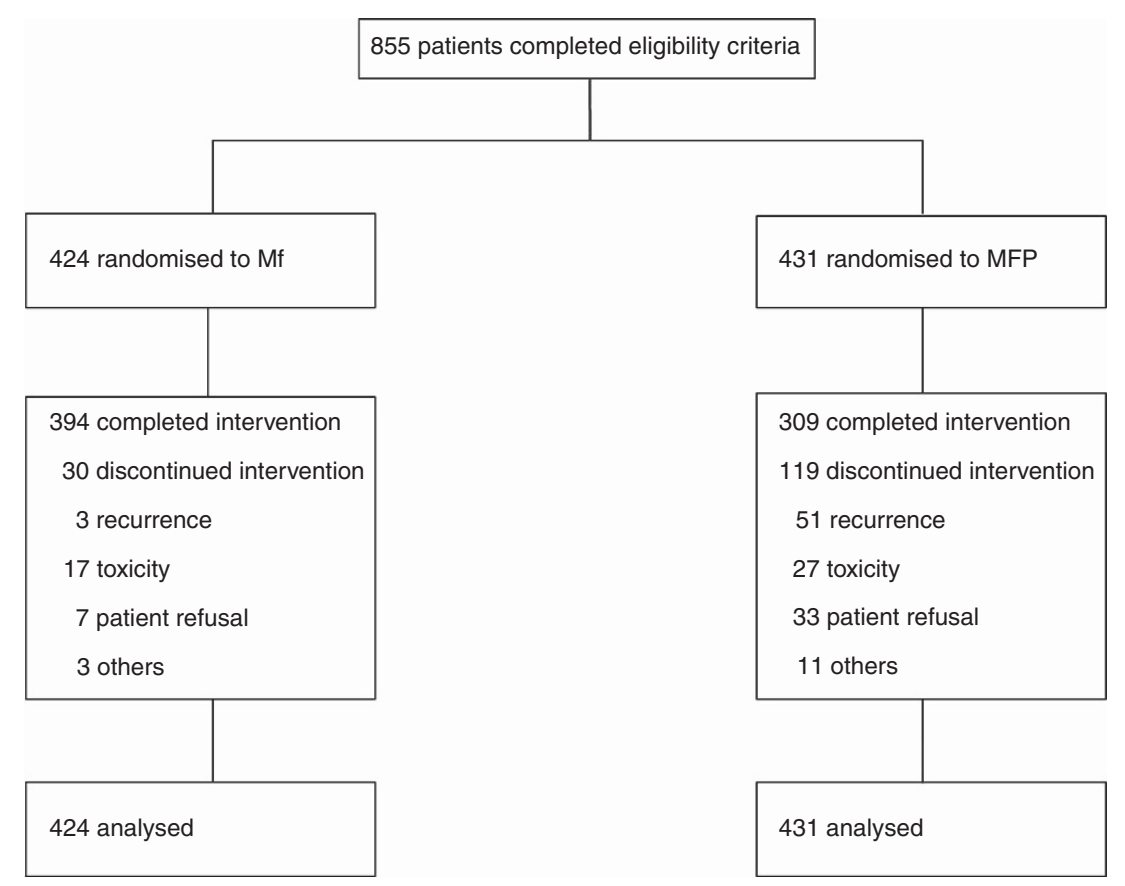

Figure 1. Trial profile. Mf, mitomycin-C plus short-term doxifluridine; MFP, mitomycin-C plus long-term doxifluridine plus cisplatin. 


\section{Table1. Baseline patient characteristics}

\begin{tabular}{|l|c|c|}
\hline Characteristics & Mf arm $(\boldsymbol{n}=\mathbf{4 2 4 )}$ & MFP arm $(\boldsymbol{n}=\mathbf{4 3 1})$ \\
\hline Age (years) & $56(29-70)$ & $55(20-70)$ \\
\hline Male gender & $294(69 \%)$ & $294(68 \%)$ \\
\hline
\end{tabular}

\section{ECOG performance status}

\begin{tabular}{|l|c|c}
\hline $0-1$ & $423(>99 \%)$ & $428(>99 \%)$ \\
2 & $1(<1 \%)$ & $3(<1 \%)$
\end{tabular}

\section{Primary site}

\begin{tabular}{|l|r|r}
\hline Proximal & $40(10 \%)$ & $33(8 \%)$ \\
Distal & $342(81 \%)$ & $351(81 \%)$ \\
Multiple/diffuse & $42(10 \%)$ & $47(11 \%)$ \\
\hline
\end{tabular}

Multiple/diffuse

\section{Type of surgery}

\begin{tabular}{l|l|l}
\hline Total gastrectomy & $162(38 \%)$ & $166(39 \%)$ \\
Subtotal gastrectomy & $262(62 \%)$ & $265(61 \%)$
\end{tabular}

\section{Lauren's classification}

\begin{tabular}{l|c|c|} 
Intestinal type & $148(35 \%)$ & $135(31 \%)$ \\
Diffuse type & $222(52 \%)$ & $235(55 \%)$ \\
Mixed type & $39(9 \%)$ & $49(11 \%)$ \\
Unknown & $15(4 \%)$ & $12(3 \%)$
\end{tabular}

\section{Pathologic T stage}

\begin{tabular}{|l|c|c|}
\hline PT1 & $9(2 \%)$ & $8(2 \%)$ \\
PT2 & $261(62 \%)$ & $235(55 \%)$ \\
PT3 & $147(35 \%)$ & $178(41 \%)$ \\
PT4 & $7(2 \%)$ & $10(2 \%)$ \\
\hline
\end{tabular}

\section{No. of lymph-node metastasis}

\begin{tabular}{|l|c|c|}
\hline 0 & $28(7 \%)$ & $43(10 \%)$ \\
$1-6$ & $256(60 \%)$ & $249(58 \%)$ \\
$7-15$ & $109(26 \%)$ & $109(25 \%)$ \\
$\geq 16$ & $31(7 \%)$ & $30(7 \%)$
\end{tabular}

\section{Overall stage $^{a}$}

\begin{tabular}{|l|c|c|}
\hline II & $220(52 \%)$ & $216(50 \%)$ \\
IIIA & $130(31 \%)$ & $136(32 \%)$ \\
IIIB & $38(9 \%)$ & $42(10 \%)$ \\
IV (M0) & $36(9 \%)$ & $37(9 \%)$
\end{tabular}

\begin{tabular}{|c|c|c|}
\hline \multicolumn{3}{|c|}{ Dose of doxifluridine } \\
\hline $460 \mathrm{mg} \mathrm{m}^{-2}$ per day & $238(56 \%)$ & $233(54 \%)$ \\
\hline $600 \mathrm{mg} \mathrm{m}^{-2}$ per day & 186 (44\%) & 198 (46\%) \\
\hline
\end{tabular}

Abbreviations: $\mathrm{Mf}=$ mitomycin-C plus short-term doxifluridine; $\mathrm{MFP}=$ mitomycin-C plus long-term doxifluridine plus cisplatin; ECOG = Eastern Cooperative Oncology Group. ${ }^{a}$ American Joint Committee on Cancer Staging System, 6th edition (2002).

per day, $P<0.001$ ), although there was no difference in cisplatin (mean 56.64 vs $56.86 \mathrm{mg} \mathrm{m}^{-2}$ per month, $P=0.67$ ).

Long-term efficacy outcomes. With a median follow-up of 6.6 years in April 2011, a total of 353 events (recurrence or death) have been observed (167 in the Mf group and 186 in the MFP group). The 3- and 5-year RFS rates were 67.0\% (95\% CI, 62.5-71.5) and $61.1 \%$ (95\% CI, 56.4-65.8) in the Mf group, respectively, and 64.9\% (95\% CI, 60.4-69.4) and 57.9\% (95\% CI, 53.2-62.6) in the MFP group, respectively. There was no statistically significant difference in RFS between the two groups (HR 1.10, 95\% CI 0.89-1.35; $P=0.39$; Figure 2A). The 3- and 5-year OS rates were $76.9 \%(95 \%$ CI, 72.9-80.9) and $66.5 \%$ (95\% CI, 62.0-71.0) in the Mf group,
Table 2. Adverse events reported by $\geq 10 \%$ of patients (safety population) ${ }^{\mathbf{a}}$

\begin{tabular}{|c|c|c|c|c|}
\hline & \multicolumn{4}{|c|}{ Toxicity grade by treatment arm } \\
\hline & \multicolumn{2}{|c|}{ Mf $\operatorname{arm}(n=422)$} & \multicolumn{2}{|c|}{ MFP arm $(n=425)$} \\
\hline & $\begin{array}{c}\text { All } \\
\text { grade }\end{array}$ & $\begin{array}{l}\text { Grade } \\
3 \text { or } 4\end{array}$ & $\begin{array}{c}\text { All } \\
\text { grades }\end{array}$ & $\begin{array}{l}\text { Grade } \\
3 \text { or } 4\end{array}$ \\
\hline \multicolumn{5}{|l|}{ Haematologic } \\
\hline $\begin{array}{l}\text { Leucopenia } \\
\text { Neutropenia } \\
\text { Anaemia } \\
\text { Thrombocytopenia }\end{array}$ & $\begin{array}{l}265(63 \%) \\
262(62 \%) \\
393(93 \%) \\
101(24 \%)\end{array}$ & $\begin{array}{c}20(5 \%) \\
47(11 \%) \\
9(2 \%) \\
14(3 \%)\end{array}$ & $\begin{array}{l}367(86 \%) \\
387(91 \%) \\
413(97 \%) \\
156(37 \%)\end{array}$ & $\begin{array}{c}24(6 \%) \\
150(35 \%) \\
16(4 \%) \\
14(3 \%)\end{array}$ \\
\hline \multicolumn{5}{|l|}{ Non-haematologic } \\
\hline $\begin{array}{l}\text { AST or ALT } \\
\text { Hyperbilirubinaemia } \\
\text { Fatigue } \\
\text { Anorexia } \\
\text { Nausea } \\
\text { Vomiting } \\
\text { Stomatitis } \\
\text { Constipation } \\
\text { Diarrhoea } \\
\text { Alopecia } \\
\text { Neuropathy } \\
\text { HFS } \\
\text { Myalgia } \\
\text { Oedema }\end{array}$ & $\begin{array}{c}119(29 \%) \\
114(27 \%) \\
343(81 \%) \\
326(77 \%) \\
293(69 \%) \\
104(25 \%) \\
77(18 \%) \\
132(31 \%) \\
219(52 \%) \\
261(62 \%) \\
149(35 \%) \\
35(8 \%) \\
109(26 \%) \\
44(10 \%)\end{array}$ & $\begin{array}{l}3(<1 \%) \\
1(<1 \%) \\
0(0 \%) \\
1(<1 \%) \\
0(0 \%) \\
7(2 \%) \\
0(0 \%) \\
1(<1 \%) \\
1(<1 \%) \\
0(0 \%) \\
0(0 \%) \\
0(0 \%) \\
0(0 \%) \\
0(0 \%)\end{array}$ & $\begin{array}{l}145(34 \%) \\
162(38 \%) \\
391(92 \%) \\
389(92 \%) \\
371(87 \%) \\
160(38 \%) \\
151(36 \%) \\
204(48 \%) \\
288(68 \%) \\
334(79 \%) \\
301(71 \%) \\
73(17 \%) \\
166(39 \%) \\
108(25 \%)\end{array}$ & $\begin{aligned} & 3(<1 \%) \\
& 4(<1 \%) \\
& 9(2 \%) \\
& 10(2 \%) \\
& 9(2 \%) \\
& 15(4 \%) \\
& 0(0 \%) \\
& 2(<1 \%) \\
& 8(2 \%) \\
& 0(0 \%) \\
& 2(<1 \%) \\
& 0(0 \%) \\
& 1(<1 \%) \\
& 0(0 \%)\end{aligned}$ \\
\hline
\end{tabular}

Abbreviations: $\mathrm{Mf}=$ mitomycin-C plus short-term doxifluridine; $\mathrm{MFP}=$ mitomycin- $\mathrm{C}$ plus long-term doxifluridine plus cisplatin; $A S T=$ aspartate aminotransferase; $A L T=$ alanine aminotransferase; HFS = hand-foot syndrome.

${ }^{\text {a }}$ Patients who did not receive allocated treatment after randomisation or were lost to follow-up after MMC administration were excluded.

respectively, and 73.1\% (95\% CI, 68.9-77.3) and 65.0\% (95\% CI, 60.5-69.5) in the MFP group, respectively. The difference in OS was also insignificant (HR 1.11, 95\% CI 0.89-1.39; $P=0.33$; Figure 2B). Median RFS and OS have not been reached in either group. In subgroup analyses for RFS (Figure 3), there was no significant interaction between study treatment and baseline characteristics. RFS and OS were not affected by whether patients were enroled before $(n=471)$ or after the protocol amendment ( $n=384 ; P=0.96$ and 0.71 , respectively). The sites of first recurrence are summarised in Table 3. Distant metastasis was more common than locoregional recurrence in both groups, and there were no significant differences between the two groups.

\section{DISCUSSION}

After a long debate, it is now globally agreed that adjuvant treatment improves survival of patients with GC who undergo curative surgery. However, there are still geographical differences in standard adjuvant treatment modalities. The intergroup-0116 (INT-0116) study of postoperative chemoradiation (CRT) (Macdonald et al, 2001), and the Medical Research Council Adjuvant Gastric Infusional Chemotherapy (MAGIC) trial of perioperative chemotherapy (Cunningham et al, 2006), demonstrated the survival benefits in AGC over surgery alone, and it has become standard adjuvant therapy in the United States and 


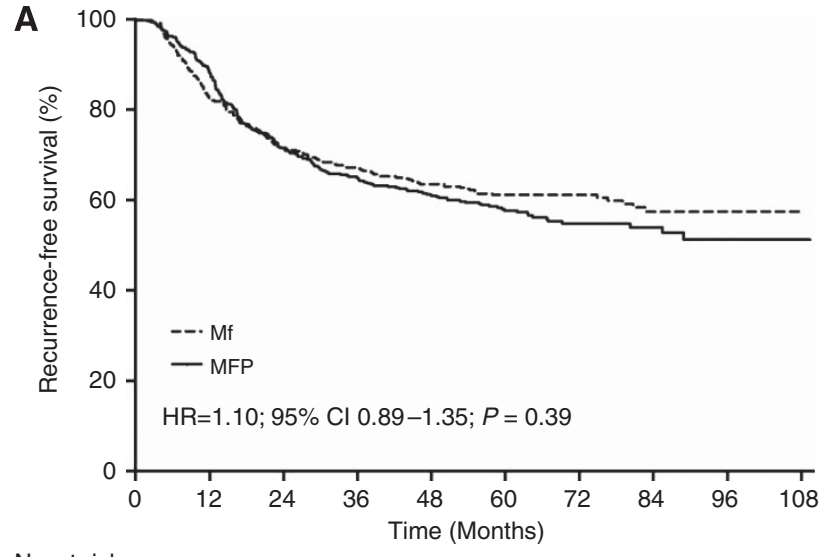

No. at risk

$\begin{array}{lllllll}\text { Mf } & 424 & 350 & 299 & 276 & 254 & 208 \\ \text { MFP } & 431 & 381 & 305 & 272 & 242 & 185\end{array}$

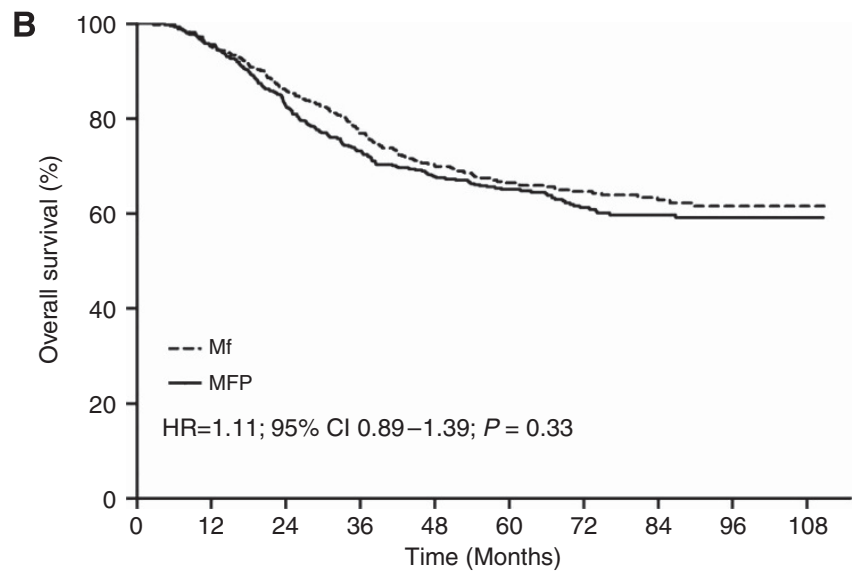

No. at risk

$\begin{array}{lllllll}\text { Mf } & 424 & 403 & 362 & 323 & 292 & 255\end{array}$

$\begin{array}{lllllll}\text { MFP } & 431 & 412 & 356 & 312 & 287 & 246\end{array}$

Figure 2. Kaplan-Meier estimates of RFS (A) and OS (B). Mf, mitomycin-C plus short-term doxifluridine; MFP, mitomycin- $C$ plus long-term doxifluridine plus cisplatin.

Europe, respectively. In Asia, where D2 surgery is a standard procedure, postoperative chemotherapy has become standard therapy on the basis of the results of the ACTS-GC (S-1 for 12 months) (Sakuramoto et al, 2007) and the recent CLASSIC trial (capecitabine and oxaliplatin for 6 months) (Bang et al, 2012). Therefore, the most relevant issue in adjuvant treatment now is how to improve the treatment outcome of current standard adjuvant treatment. In this context, the results of this study offer timely suggestions regarding the question.

The control treatment of this study, Mf regimen is a reasonable adjuvant chemotherapy for which benefit over no treatment was confirmed by the recent GASTRIC meta-analysis study (GASTRIC Group, 2010). This study was reasonably powered to assess the clinically relevant improvement in RFS, and follow-up period was sufficient to confirm the long-term outcomes. Nevertheless, the RFS and OS curves of the two arms overlapped and there was no trend of benefit in the MFP arm. This strongly indicates that simple intensification of the adjuvant chemotherapy with Mf regimen by the addition of cisplatin and prolongation of doxifluridine administration is not effective. We do not know why this strategy is not working in the adjuvant setting, while it is working in the palliative setting. A meta-analysis showed that combination chemotherapy is better than single agent, mainly 5-
FU, and the Japanese SPIRITS trial demonstrated that addition of cisplatin to fluoropyrimidine could improve the survivals in patients with metastatic or recurrent GC (Wagner et al, 2006; Koizumi et al, 2008).

Many of the adjuvant chemotherapy trials testing polychemotherapeutic agents with cisplatin failed to achieve survival benefit over reference treatment (Chipponi et al, 2004; Bouche et al, 2005; Cascinu et al, 2007; Di Costanzo et al, 2008). These failures may result from low statistical power of the studies due to their small size, but low completion rates owing to toxicity also played an important role in poor performance. In our study, we gave $60 \mathrm{mg} \mathrm{m}^{-2}$ of cisplatin to obtain a commonly prescribed dose of cisplatin in East Asia for lower toxicities and better compliance. This strategy resulted in better tolerability, but additive survival benefit was not obtained.

This result is in line with the recent results of CALGB 80101 and ITACA-S trials (Fuchs et al, 2011; Bajetta et al, 2012). In CALGB 80101 study, adjuvant CRT for gastric or gastro-oesophageal junction adenocarcinoma using epirubicin, cisplatin and 5-FU (ECF) was compared with bolus 5-FU/LV before and after 5-FU/ radiotherapy, but the ECF arm did not achieve better disease-free survival (HR, 1.00, 95\% CI, 0.79-1.27; $P=0.99$ ) and OS (HR, 1.03, 95\% CI, 0.80-1.34; $P=0.80$ ) (Fuchs et al, 2011). Furthermore, ITACA-S trial failed to demonstrate the enhanced efficacy of intensified chemotherapy (four cycles of 5-FU, LV and irinotecan, followed by three cycles of docetaxel and cisplatin) compared with control chemotherapy (nine cycles of 5-FU and LV) in terms of disease-free survival (HR, 0.98, 95\% CI, 0.83-1.16; $P=0.83$ ) and OS (HR, 1.0, 95\% CI, 0.83-1.20; $P=0.97$ ) (Bajetta et al, 2012).

In AMC0101 study (Kang et al, 2008), a companion adjuvant chemotherapy trial of AMC0201, two more strategies, intraperitoneal chemotherapy and early start of chemotherapy, were applied in GC patients with macroscopically recognisable serosa invasion compared with the AMC0201 study. Moreover, addition of these four strategies to $\mathrm{Mf}$ chemotherapy resulted in a significantly improved RFS and OS, while addition of two strategies, prolonged doxifluridine and addition of cisplatin were not effective in AMC0201. It therefore appears that it is not the simple intensification by additional chemotherapeutic agents or prolongation of duration of chemotherapy, but rather the new strategies of early initiation of systemic chemotherapy and/or intraperitoneal chemotherapy that can be effective strategies to further improve the current standard adjuvant therapy. We believe these strategies should be tested in future clinical trials. Neoadjuvant chemotherapy, which is the earliest adjuvant chemotherapy we can have, is currently investigated in PRODIGY (NCT01515748) and JCOG0501 (C000000279) trials. These should provide findings concerning the efficacy of neoadjuvant chemotherapy in patients who will receive D2 gastrectomy and postoperative S-1.

As mentioned above, geographic differences exist in standard adjuvant treatments, in terms of therapeutic modality, timing of treatment and chemotherapy regimens. These discrepancies may be influenced by global differences in standard surgical methods or study populations. In Asia where D2 gastrectomy is the standard surgery, postoperative oral fluoropyrimidine-based adjuvant chemotherapy, such as S-1 for 1 year and capecitabine plus oxaliplatin for 6 months, is a proved treatment option for localised GC. However, because there has been lack of randomised trial comparing these regimens, it is difficult to determine which one is superior to others.

During the study, study protocol was amended in regards to sample size and dose of doxifluridine based on the results of interim analysis. The interim analysis was originally planned for safety monitoring and potential sample size recalculation based on the distribution of pathological stage in study population. Since this study started, relatively low dose of doxifluridine had been 


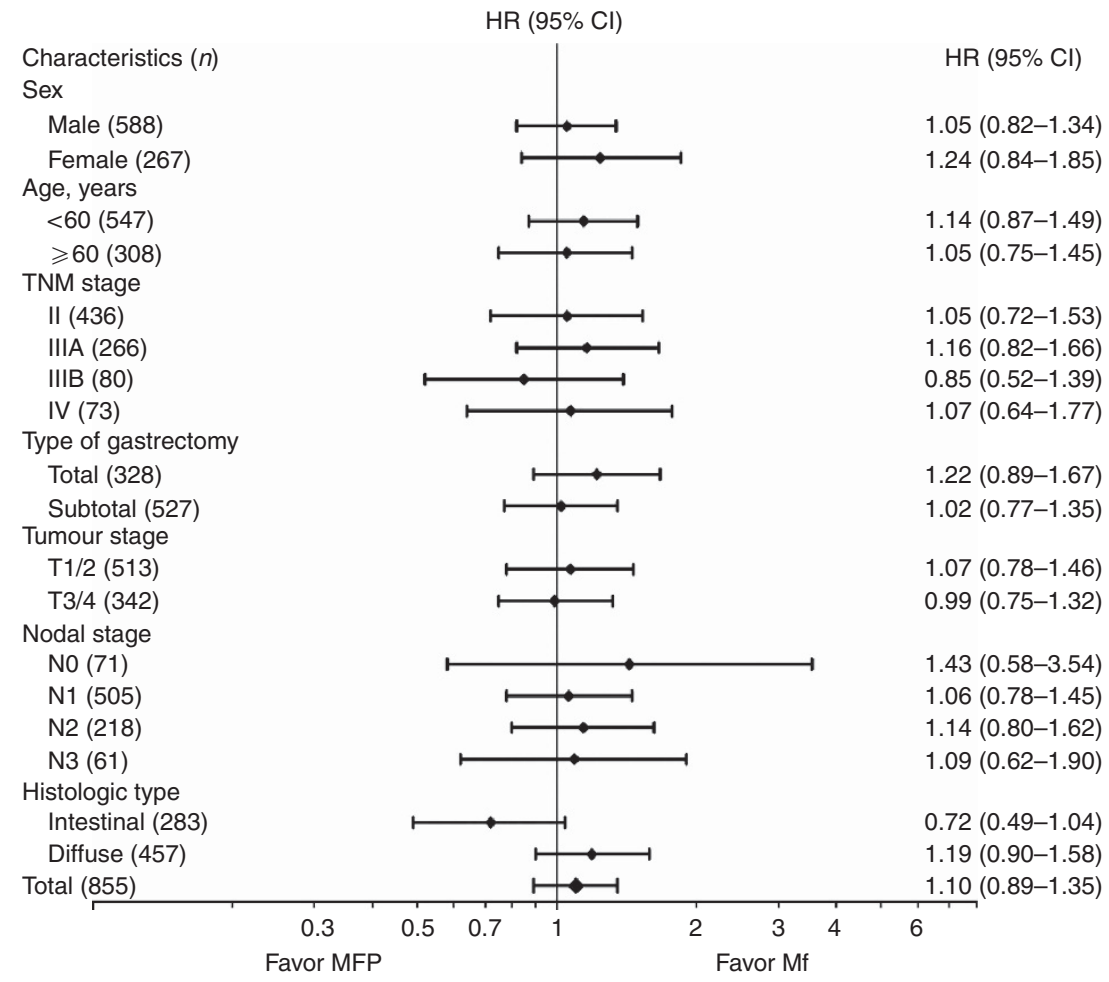

Figure 3. Subgroup analysis for RFS. Mf, mitomycin-C plus short-term doxifluridine; MFP, mitomycin-C plus long-term doxifluridine plus cisplatin; $\mathrm{HR}$, hazard ratio; $\mathrm{Cl}$, confidence interval.

\begin{tabular}{|c|c|c|c|}
\hline Site of recurrence & $\begin{array}{l}\text { Mf arm } \\
(n=424)\end{array}$ & $\begin{array}{l}\text { MFP arm } \\
(n=431)\end{array}$ & $\boldsymbol{P}$-value \\
\hline Total no. of recurrence & $158(37 \%)$ & 170 (39\%) & \\
\hline Locoregional & $28(7 \%)$ & $39(9 \%)$ & 0.20 \\
\hline Distant & $130(31 \%)$ & $131(30 \%)$ & 0.94 \\
\hline Haematogenous & $61(14 \%)$ & 75 (17\%) & 0.26 \\
\hline Peritoneal & 65 (15\%) & 55 (13\%) & 0.32 \\
\hline Lymphatic & $46(11 \%)$ & 44 (10\%) & 0.82 \\
\hline \multicolumn{4}{|c|}{$\begin{array}{l}\text { Abbreviations: } M f=\text { mitomycin- } C \text { plus short-term doxifluridine; } M F P=\text { mitomycin-C plus } \\
\text { long-term doxifluridine plus cisplatin. } \\
{ }^{a} \text { More than one recurrent site in some patients. }\end{array}$} \\
\hline
\end{tabular}

indicated by several participating investigators. Because the safety monitoring at interim analysis showed mild and tolerable toxicity profile of original dose, we decided to increase the doxifluridine dose from 460 to $600 \mathrm{mg} \mathrm{m}^{-2}$ per day. Although actual administration of doxifluridine was increased without significant aggravation of toxicity except neutropenia, there was no difference in survival outcomes between patients enroled before and after the introduction of a protocol amendment to increase daily dose of doxifluridine. The assumption of RFS in control arm was based on our previous results with Mf chemotherapy. However, the RFS of the control arm should change according to the stage distribution of the study population since stage is the most important prognostic factor. We wanted to check if the stage distribution of the study population were as we had assumed before. And, if there were differences in the interim analysis, we wanted to reassume the RFS of the control arm based on this stage distribution of the study population, and subsequently the sample size required to detect the efficacy of the experimental arm. Actually, because the greater number of patients with early stage was enroled than we originally expected, we had to re-estimate the expected 3-year RFS of control arm and recalculate the sample size in the planned interim analysis. Although this required increasing the sample size, rapid patient accrual enabled us to detect even small differences in efficacy between treatment groups.

In conclusion, prolongation of doxifluridine administration and addition of cisplatin to adjuvant chemotherapy with MMC plus 3 months of doxifluridine could be safely performed but did not improve the treatment outcome in curatively resected GC patients.

\section{ACKNOWLEDGEMENTS}

This study was presented in part at the 44th American Society of Clinical Oncology (ASCO) annual meeting, 30 May-3 June 2008 in Chicago, IL, USA. Extended analysis of the study was presented at the 36th European Society of Medical Oncology (ESMO) annual congress, 23-27 September 2011 in Stockholm, Sweden and the 2012 Gastrointestinal Cancers Symposium, 19-21 January 2012 in San Francisco, CA, USA. The authors are indebted to Professor J Patrick Barron, Chairman of the Department of International Medical Communications of Tokyo Medical University, for his pro bono review of this manuscript.

\section{REFERENCES}

Bajetta E, Floriani I, Di Bartolomeo M, Labianca R, Landi L, Santoro A, Casaretti R, Pasquini E, Di Fabio F, Rondini E, Pinotti G, Bidoli P, Rosati G, Mambrini A, Ciarlo A, Cordio SS, Ricci S, Frassineti L, Di Costanzo F, Bochicchio AM (2012) Intergroup Trial of Adjuvant Chemotherapy in Adenocarcinoma of the Stomach (ITACA-S) trial: Comparison of a sequential treatment with irinotecan (CPT-11) plus 5-fluorouracil (5-FU)/folinic acid (LV) followed by docetaxel and cisplatin 
versus a $5-\mathrm{FU} / \mathrm{LV}$ regimen as postoperative treatment for radically resected gastric cancer. ASCO Meet Abstr 30: LBA4001.

Bang YJ, Kim YW, Yang HK, Chung HC, Park YK, Lee KH, Lee KW, Kim YH, Noh SI, Cho JY, Mok YJ, Ji J, Yeh TS, Button P, Sirzen F, Noh SH (2012) Adjuvant capecitabine and oxaliplatin for gastric cancer after D2 gastrectomy (CLASSIC): a phase 3 open-label, randomised controlled trial. Lancet 379(9813): 315-321.

Bouche O, Ychou M, Burtin P, Bedenne L, Ducreux M, Lebreton G, Baulieux J, Nordlinger B, Martin C, Seitz JF, Tigaud JM, Echinard E, Stremsdoerfer N, Milan C, Rougier P (2005) Adjuvant chemotherapy with 5-fluorouracil and cisplatin compared with surgery alone for gastric cancer: 7-year results of the FFCD randomized phase III trial (8801). Ann Oncol 16: 1488-1497.

Cascinu S, Labianca R, Barone C, Santoro A, Carnaghi C, Cassano A, Beretta GD, Catalano V, Bertetto O, Barni S, Frontini L, Aitini E, Rota S, Torri V, Floriani I, Pozzo C, Rimassa L, Mosconi S, Giordani P, Ardizzoia A, Foa P, Rabbi C, Chiara S, Gasparini G, Nardi M, Mansutti M, Arnoldi E, Piazza E, Cortesi E, Pucci F, Silva RR, Sobrero A, Ravaioli A (2007) Adjuvant treatment of high-risk, radically resected gastric cancer patients with 5-fluorouracil, leucovorin, cisplatin, and epidoxorubicin in a randomized controlled trial. J Natl Cancer Inst 99: 601-607.

Chang HM, Jung KH, Kim TY, Kim WS, Yang HK, Lee KU, Choe KJ, Heo DS, Bang YJ, Kim NK (2002) A phase III randomized trial of 5-fluorouracil, doxorubicin, and mitomycin $\mathrm{C}$ versus 5 -fluorouracil and mitomycin $\mathrm{C}$ versus 5-fluorouracil alone in curatively resected gastric cancer. Ann Oncol 13: $1779-1785$.

Chang HM, Kang YK, Min YJ, Zang DY, Kim GY, Yang DH, Jang SJ, Yook JH, Oh ST, Kim BS (2008) A randomized phase III trial comparing mitomycin-C plus short-term doxifluridine (Mf) versus mitomycin-C plus long-term doxifluridine plus cisplatin (MFP) after curative resection of advanced gastric cancer (AMC 0201) (NCT00296335). J Clin Oncol 26(Suppl):abstr 4531.

Chipponi J, Huguier M, Pezet D, Basso N, Hay JM, Quandalle P, Jaeck D, Fagniez PL, Gainant A (2004) Randomized trial of adjuvant chemotherapy after curative resection for gastric cancer. Am J Surg 187: 440-445.

Cirera L, Balil A, Batiste-Alentorn E, Tusquets I, Cardona T, Arcusa A, Jolis L, Saigi E, Guasch I, Badia A, Boleda M (1999) Randomized clinical trial of adjuvant mitomycin plus tegafur in patients with resected stage III gastric cancer. J Clin Oncol 17: 3810-3815.

Cunningham D, Allum WH, Stenning SP, Thompson JN, Van de Velde CJ, Nicolson M, Scarffe JH, Lofts FJ, Falk SJ, Iveson TJ, Smith DB, Langley RE, Verma M, Weeden S, Chua YJ. MAGIC Trial Participants (2006)

Perioperative chemotherapy versus surgery alone for resectable gastroesophageal cancer. N Engl J Med 355: 11-20.

Di Costanzo F, Gasperoni S, Manzione L, Bisagni G, Labianca R, Bravi S, Cortesi E, Carlini P, Bracci R, Tomao S, Messerini L, Arcangeli A, Torri V, Bilancia D, Floriani I, Tonato M, Dinota A, Strafiuso G, Corgna E, Porrozzi S, Boni C, Rondini E, Giunta A, Monzio Compagnoni B, Biagioni F, Cesari M, Fornarini G, Nelli F, Carboni M, Cognetti F, Enzo MR, Piga A, Romiti A, Olivetti A, Masoni L, De Stefanis M, Dalla Mola A, Camera S, Recchia F, De Filippis S, Scipioni L, Zironi S, Luppi G, Italia M, Banducci S, Pisani Leretti A, Massidda B, Ionta MT, Nicolosi A, Canaletti R, Biscottini B, Grigniani F, Rovei R, Croce E, Carroccio R, Gilli G, Cavalli C, Olgiati A, Pandolfi U, Rossetti R, Natalini G, Foa P, Oldani S, Bruno L, Cascinu S, Catalano G, Catalano V, Lungarotti F, Farris A, Sarobba MG, Trignano M, Muscogiuri A, Francavilla F, Figoli F, Leoni M, Papiani G, Orselli G, Antimi M, Bellini V, Cabassi A, Contu A, Pazzola A, Frignano M, Lastraioli E, Saggese M, Bianchini D, Antonuzzo L, Mela M, Camisa R (2008) Adjuvant chemotherapy in completely resected gastric cancer: a randomized phase III trial conducted by GOIRC. J Natl Cancer Inst 100: 388-398.

Earle CC, Maroun JA (1999) Adjuvant chemotherapy after curative resection for gastric cancer in non-Asian patients: revisiting a meta-analysis of randomised trials. Eur J Cancer 35: 1059-1064.

Fuchs CS, Tepper JE, Niedzwiecki D, Hollis D, Mamon HJ, Swanson R, Haller DG, Dragovich T, Alberts SR, Bjarnason GA, Willett CG, Enzinger PC, Goldberg RM, Venook AP, Mayer RJ (2011) Postoperative adjuvant chemoradiation for gastric or gastroesophageal junction (GEJ) adenocarcinoma using epirubicin, cisplatin, and infusional (CI) 5-FU (ECF) before and after CI 5-FU and radiotherapy (CRT) compared with bolus 5-FU/LV before and after CRT: Intergroup trial CALGB 80101. ASCO Meet Abstr 29: 4003.

GASTRIC Group (2010) Benefit of adjuvant chemotherapy for resectable gastric cancer: a meta-analysis. JAMA 303: 1729-1737.

Hermans J, Bonenkamp JJ, Boon MC, Bunt AM, Ohyama S, Sasako M, Van de Velde CJ (1993) Adjuvant therapy after curative resection for gastric cancer: meta-analysis of randomized trials. J Clin Oncol 11: 1441-1447.

Jemal A, Bray F, Center MM, Ferlay J, Ward E, Forman D (2011) Global cancer statistics. CA Cancer J Clin 61: 69-90.

Kang YK, Change HM, Zang DY, Lee JL, Kim TW, Yang DH, Jang SJ, Yook JH, Oh ST, Kim BS (2008) Postoperative adjuvant chemotherapy for grossly serosa-positive advanced gastric cancer: A randomized phase III trial of intraperitoneal cisplatin and early mitomycin-C plus long-term doxifluridine puls cisplatin (iceMFP) versus mitomycin-C plus short-term doxifluridine (Mf) (AMC0101) (NCT00296322). ASCO Meet Abstr 26: LBA4511.

Koizumi W, Narahara H, Hara T, Takagane A, Akiya T, Takagi M, Miyashita K, Nishizaki T, Kobayashi O, Takiyama W, Toh Y, Nagaie T, Takagi S, Yamamura Y, Yanaoka K, Orita H, Takeuchi M (2008) S-1 plus cisplatin versus S-1 alone for first-line treatment of advanced gastric cancer (SPIRITS trial): a phase III trial. Lancet Oncol 9: 215-221.

Koo DH, Lee JL, Kim TW, Chang HM, Ryu MH, Yook JH, Oh ST, Kim BS, Lee JS, Kang YK (2007) Adjuvant chemotherapy with 5-fluorouracil, doxorubicin and mitomycin-C (FAM) for 6 months after curative resection of gastric carcinoma. Eur J Surg Oncol 33: 843-848.

Macdonald JS, Smalley SR, Benedetti J, Hundahl SA, Estes NC, Stemmermann GN, Haller DG, Ajani JA, Gunderson LL, Jessup JM, Martenson JA (2001) Chemoradiotherapy after surgery compared with surgery alone for adenocarcinoma of the stomach or gastroesophageal junction. $N$ Engl J Med 345: 725-730.

Nakajima T, Ota K, Ishihara S, Oyama S, Nishi M, Hamashima N (1994) Meta-analysis of 10 postoperative adjuvant chemotherapies for gastric cancer in CIH. Gan To Kagaku Ryoho 21: 1800-1805.

Sakuramoto S, Sasako M, Yamaguchi T, Kinoshita T, Fujii M, Nashimoto A, Furukawa H, Nakajima T, Ohashi Y, Imamura H, Higashino M, Yamamura Y, Kurita A, Arai K (2007) Adjuvant chemotherapy for gastric cancer with S-1, an oral fluoropyrimidine. N Engl J Med 357: 1810-1820.

Wagner AD, Grothe W, Haerting J, Kleber G, Grothey A, Fleig WE (2006) Chemotherapy in advanced gastric cancer: a systematic review and metaanalysis based on aggregate data. J Clin Oncol 24: 2903-2909.

This work is published under the standard license to publish agreement. After 12 months the work will become freely available and the license terms will switch to a Creative Commons AttributionNonCommercial-Share Alike 3.0 Unported License.

Supplementary Information accompanies this paper on British Journal of Cancer website (http://www.nature.com/bjc) 